\title{
ON Berkeley's Solution to the Barrovian CASE
}

\author{
Carlos Alberto Cardona \\ Universidad del Rosario (Bogotá), ColOMBIA \\ carlos.cardona@urosario.edu.co \\ JULIANA GUTIÉRREZ \\ Universidad del Rosario (Bogotá) and Universidad de los Andes (Bogotá), ColomBiA \\ j.gutierrezv@uniandes.edu.co
}

\begin{abstract}
At the beginning of the $18^{\text {th }}$ century, Berkeley believed an anomaly pointed out by Isaac Barrow could be regarded as important evidence against the optical theories that had been established and standardized thanks to the works of Kepler and Newton. In this article, we want to show that Berkeley's treatment of the Barrovian Case does not falsify these theories. We will contend that the strategy used by Berkeley to resolve the anomaly by alluding to a change of convention is a strategy that the classical theorist could use as well. In other words, the classical theorist could also appeal to a change of convention to deal with the problem.
\end{abstract}

Keywords: Standard divergence $\bullet$ anomalous divergence $\bullet$ geometrical theory $\bullet$ crucial anomaly $\bullet$ perception of distance

\section{Introduction}

Europe entered the $18^{\text {th }}$ century with powerful research programs in development. Copernicus's heliocentrism had been established as a new paradigm; Newton's mechanics appeared to be a program with the capacity to unify the study of natural forces; the invention of calculus was opening new doors in mathematics; and optics, thanks to the synthesis of Kepler, was emerging as a mature science. The academic setting in Ireland was not far from the optimism that this new research environment was providing. George Berkeley was an insightful witness of the technical contributions of such programs and, at the same time, a severe critic of their foundations.

The Commentaries, probably written between 1707 and 1708, were preliminary notes and suggestions for the presentation of a new philosophical system and a new research program that the author had in mind. This new system came out in two major works, Essay towards a New Theory of Vision (NTV) in 1709 and A Treatise Concerning the Principles of Human Knowledge (PHK) in $1710 .{ }^{1}$ Berkeley dedicated an important portion of his philosophical work to a powerful attack on abstract ideas. 
This attack led him to defend, among other things, some sort of immaterialism ( $P H K$, $\S \S 68-84)$, to reject the notions of absolute time and space defended by Newton (PHK, $\S 97-117)$, to criticize the alleged existence of infinitesimal magnitudes (PHK, §118133), and to uncover the absurdity of the principles of the new optics (NTV). ${ }^{2}$

The criticisms of the new optics, mainly gathered in NTV and The Theory of Vision Vindicated and Explained (TVV), published in 1733, focused on showing the unintelligibility of the methods suggested by the geometrical theory of vision (from here on, geometrical theory) to explain the way we perceive the distance, size, and disposition of objects. This theory assumed that, through the perception of lines and angles and, moreover, with help of a geometrical calculus (which may well be unconscious), the subject manages to give an account of such characteristics of objects. However, for the Irish philosopher, this made no sense. Berkeley asked his readers to take a good look and evaluate if they indeed had any awareness of seeing rays in their visual field or of measuring angles (NTV, $\S \S 12-15,19,22,52,90)$. Because this exam leads to a negative answer, it was hoped that the reader would begin to suspect the arguments of the geometrical theory.

The argumentative structure in NTV can be seen as being composed of two different sections, a negative section and a positive section. In the negative section, Berkeley defended that we do not perceive solely through our sense of sight the distance between an object and the observer. In the positive section, Berkeley showed that the objective tactile distance between an object and observer could be associated with certain visual perceptions by virtue of the constant repetition of successful experiences of correlation. That being the case, visual information could be considered as a sign that helps us anticipate possible tactile sensations. ${ }^{3}$ Hence, this philosopher advocated for a semiotic approach for vision. Keeping this in mind, the strategy for defending the unintelligibility of the visual perception of the distance between the observer and the object, its size and disposition follows the same pattern: (i) Berkeley first shows serious difficulties in the geometrical theory, offering arguments for questioning its basic principles; (ii) he then shows that these difficulties can be solved if we admit that the objects that belong solely to our visual field are associated, through contingent bonds, with the objects of our sense of touch; (iii) he finally suggests a theory that conceives the process of visually perceiving something as if it was analogous to interpreting a language. In this article, we focus on some of the passages that are intended to show the limitations of the geometrical theory in explaining the perception of distance. We thus concentrate exclusively on the role that the difficulty pointed out by Isaac Barrow (1630-1677) played in the formulation of Berkeley's criticism.

In the negative section of NTV, Berkeley presented several arguments for rejecting the view that distance belonged to the proper sensibles of vision. Distance, understood as the line between the eye and the object that is being observed, always 
projects a point on the retina regardless of the length of that line (§3). Therefore, if we manage to estimate how close or far from us an object is from our reach, it is not because we perceive it directly but because we associated with another idea that is, in itself, directly perceived. Given that we do not apprehend angles or lines aided solely by our vision, these cannot be the path for perceiving the idea of distance. We need more information (tactile information) to be able to perceive it. With these arguments, Berkeley intended to show that claiming we perceive distance solely through vision is absurd and unintelligible.

To strengthen his criticism, Berkeley tried to show that the geometrical theory was incapable of solving the anomaly stated by Isaac Barrow while his semiotic approach was able to explain the anomaly. Berkeley hoped Barrow's problem could be regarded as a crucial anomaly that falsified the geometrical theory and ruled in favor of his own theory: "This phenomenon [the Barrovian case] as it entirely subverts the opinion of those, who will have us judge of distance by lines and angles, on which supposition it is altogether inexplicable, so it seems to me no small confirmation, of the truth of that principle whereby it is explained [Berkeley's own theory]." (NTV, §33) However, in this article we intend to show that Barrow's difficulty cannot be considered as a crucial anomaly. We will argue that Berkeley's strategy for facing this difficulty with principles from his theory could also be used, with the same right, with the principles of the geometrical theory. We are not arguing in favor of the geometrical theory; we merely want to show that Berkeley's use of Barrow's problem is not a conclusive argument against it. With this goal in mind, the article has the following structure. First, we present the main principles of the geometrical theory. Second, we explain the anomaly pointed out by Barrow. Third, we lay out Berkeley's solution to the problem. Finally, we show how Berkeley's strategy of solving this difficulty can also be used with the aid of the principles of the geometrical theory.

\section{Basic principles of the geometrical theory}

Colin Murray Turbayne, in his book The Myth of Metaphor (1962) (where he, inspired by Berkeley, offers a program that conceives our visual perception as a language) presents five principles that summarize the basic assumptions of the geometrical paradigm of visual perception (pp.141-58). We will adopt, with some small changes, the five principles laid out by Turbayne to characterize the geometrical theory. Each principle will be referred to with the notation $P_{n}$.

First principle $\left(P_{1}\right)$. The beams of light that diverge from each point of an object and are forced, with the intervention of an optical instrument, to gather at a single point on a pictoric veil, will produce an inverted image of the object on that veil. Kepler was one of the first to call such pictoric images "real images". ${ }^{4}$ The simplest 
optical instrument that we can imagine is a camera obscura with an aperture reduced to a point. Figure 1 shows the object $A B$ facing a camera obscura with an opening $O$ and its real image $A^{\prime} B^{\prime}$. The real image (or painting) of the object $A B$ can be obtained if all the light rays coming from each point of the object are forced to converge at single points on a pictoric veil. If the optical instrument consists on a lens or mirror, the success or failure of obtaining a more precise convergence on the same pictoric veil depends on more complex circumstances. ${ }^{5}$

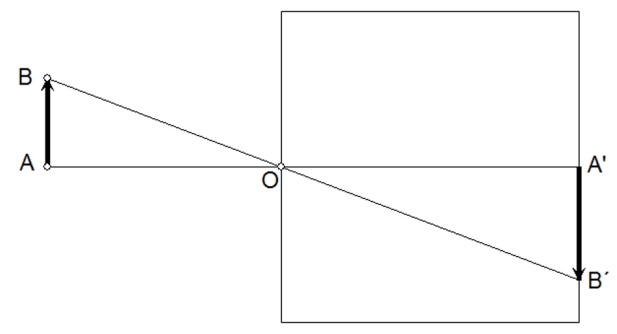

Figure 1: Formation of real images (or paintings) [https://www.geogebra.org/m/czeuxtjb. Dynamic points: $\mathrm{A}$ and $\mathrm{B}]{ }^{6}$

Second principle $\left(P_{2}\right)$. The eye resembles a camera obscura whose aperture (which is small, but not as small as a point) has a complex system of lenses. The geometrical properties of this system slightly change to allow the formation of a clear painting of the object to which we address our attention on the back of the camera, which is the place where the retina lies. This principle sums up Kepler's most important contribution to the geometrical theory.

Third and fourth principle $\left(P_{3}\right.$ and $\left.P_{4}\right)$. The third principle establishes that we can evaluate the distance of external objects through a triangulation that demands a primitive familiarity with the distance between our eyes, and, second, some sort of recognition of the angles formed by the main rays that come from a representative point of the object and are directed towards each of our eyes. Kepler introduces this principle in the following way: "since to each animal a pair of eyes is given by nature, with a certain distance between them, by this support the sense of vision is most rightly used to judge the distance of Visibles, provided that distance has a perceptible ratio to the distance of the eyes" (2000[1604], p.79). If $O_{1}$ and $O_{2}$ represent the location of the eyes separated by the distance $\mathrm{d}$ (Figure 2) and $\alpha$ and $\beta$ are the angles that the sensory apparatus apprehends in relation to the direction in which each eye contemplates the object $A$, a simple trigonometrical evaluation allows us to estimate the angle $O_{1} A O_{2}$ and the distances $O_{1} A$ and $O_{2} A$. As you can see, the angle $O_{1} A O_{2}$ is greater when the object is closer. The fourth principle ascertains that in the case for monocular vision, instead of considering the distance between the two eyes, 
the observer considers the width of the pupil in order to judge distance. Following figure $2, \mathrm{O}_{1} \mathrm{O}_{2}$ would represent the width of the pupil.

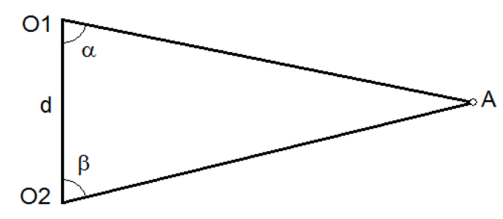

Figure 2: Judgement of distance [https://www.geogebra.org/m/seqzvrk4 Dynamic points: $O_{1}$, $\mathrm{O}_{2}$ and $\left.A\right]$

Fifth principle $\left(P_{5}\right)$. An object seen by reflection or refraction is seen in the place from where the rays seem to diverge in their entrance to the eye. In Kepler's words, "the genuine place of the image is that point in which the visual rays from the two eyes meet, extended through their respective points of refraction or reflection" (2000[1604], p.85). ${ }^{7}$ Figure 3 shows, according to this principle, the place where an object seen through an optical instrument is perceived. The upper point $A$ of the object seen through a plane mirror (above) or a lens (below) is seen at point $A^{\prime}$. The three rays drawn from $A$ arrive to the eye at points $C, D$, and $E$ after reflection or refraction. ${ }^{8}$
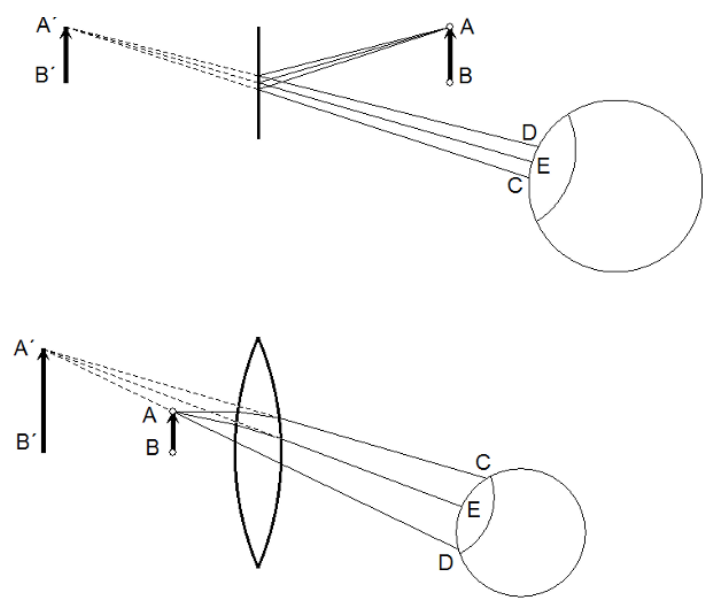

Figure 3: Situation of objects perceived with the intervention of optical instruments [https: //www.geogebra.org/m/ypamchsw Dynamic points: $A, B$ and the center of the eye. In addition, the reader can manipulate the eye's size [Eye] and the pencil's amplitude $[\alpha]]$ 


\section{Barrovian Case}

Berkeley sought to weaken the geometrical theory by showing that it was unable to explain an experiential difficulty presented by Isaac Barrow at the end of his lectures on Optics; this difficulty is known as the Barrovian Case. Berkeley hoped to present this anomaly as a crucial anomaly; for him, the Barrovian Case showed that the principles of the geometrical theory are insufficient for explaining our perception of distance. Added to this negative argument, Berkeley also expected to achieve victory by presenting his theory as successful in explaining this difficulty. Barrow characterized this finding as follows: "before I quit it for good and all, the fair and ingenuous dealing that I owe both to you and to truth obligeth me to acquaint you with a certain ontoward difficulty, which seems directly opposite to the doctrine I have been hitherto inculcating, at least, admits of no solution from it" (1860[2013], p.152).

Unfortunately, many scholars either omit or misunderstand Berkeley's allusion to the Barrovian Case. Pitcher (1977) and Stack (1991) ignore the problem, while Tipton (1974, pp.207-8) merely mentions the problem without giving any detail. Luce (1934) does highlight the importance of Barrow's work in Berkeley's discussions (especially with mathematicians) but does not deal with the technical details of the difficulty. Moreover, Armstrong (1960, p.19) does not focus on it, and Margaret Atherton (1990, pp.86-8) presents it as one (among many) of the arguments that Berkeley offers against the geometrical theory but does not evaluate whether it is a successful argument or not. For us, however, omitting the details of the case prevents us from fully understanding Berkeley's argument and the way he uses this anomaly.

Meanwhile, C. M. Turbayne faces the problem and regards the technical aspects as important because he is interested in seeing the Barrovian Case precisely as the exhibition of a crucial anomaly whose empirical results rule in favor of Berkeley and against the defenders of the geometrical theory: "the Barrovian Case is a disconfirming instance of the Geometrical Theory considered as a theory designed to explain how we see" (1962, p.177). Furthermore, T. Lennon deals with all the details and, unlike Turbayne, tries to demonstrate that the supporters of the geometrical theory could solve the difficulty and, therefore, the case should not be seen as a crucial anomaly in favor of Berkeley's theory: "The Barrovian Case is a test case for Berkeley, not as an experimentum crucis that adjudicates between his view and his competitors', but as a phenomenon that should be, and in fact is, successfully accommodated by them all." (2007, p.52). In the present article we will argue, in accordance with Lennon, that Berkeley's strategy for conceiving a way out of the difficulty can be used from the perspective of the geometrical theory.

Let us now focus on the problem. We will quote the long final passages from 
Barrow's conferences on Optics (just as Berkeley does), where Barrow introduces the difficulty. The presentation will refer to figure 4:

Before the double convex glass or concave speculum $E B F$, let the point $A$ be placed at such a distance that the rays proceeding from $A$, after refraction or reflexion, be brought to unite somewhere in the $A x A B$. And suppose the point of union (i.e. the image of the point $A[\ldots]$ ) to be $Z$; between which and $B$, the vertex of the glass or speculum, conceive the eye to be anywhere placed. The question now is, where the point $A$ ought to appear? Experience shews that it does not appear behind at the point $Z$, and it were contrary to nature that it should, since all the impression which affects the sense comes from towards A. But from our tenets it should seem to follow that it would appear before the eye at a vast distance off, so great as should in some sort surpass all sensible distance. For since if we exclude all anticipations and prejudices, every object appears by so much the farther off, by how much the rays it sends to the eye are less diverging. And that object is thought to be most remote from which parallel rays proceed unto the eye. Reason would make one think that object should appear at yet a greater distance which is seen by converging rays. Moreover it may in general be asked concerning this case what it is that determines the apparent place of the point $A$, and maketh it to appear after a constant manner sometimes nearer, at other times farther off? To which doubt I see nothing that can be answered agreeable to the principles we have laid down except only that the point $A$ ought always to appear extremely remote. But on the contrary we are assured by experience that the point A appears variously distant, according to the different situations of the eye between the points $A$ and $Z$. And that it doth [almost] never (if at all) seem farther off, than it would if it were beheld by the naked eye, but on the contrary it doth sometimes appear much nearer. Nay, it is even certain that by how much the rays falling on the eye do more converge by so much the nearer doth the object seem to approach. For the eye being placed close to the point $B$, the object $A$ appears nearly in its own natural place, if the point $B$ is taken in the glass, or at the same distance, if in the speculum. The eye being brought back to $O$, the object seems to draw near: and being come to $P$ it beholds it still nearer. And so on little and little, till at length the eye being placed somewhere, suppose at $Q$, the object appearing extremely near, begins to vanish into mere confusion. All which doth seem repugnant to our principles, at least not rightly to agree with them. Nor is our tenet alone struck at by this experiment, but likewise all others that ever came to my knowledge are, every whit as much, endangered by it. The ancient one especially (which is most commonly received, and comes nearest to mine) seems to be so effectually overthrown thereby that the most learned Tacquet has been forced to reject that principle, as false and uncertain, on which alone he had built almost his whole Catoptrics; and consequently by taking away the foundation, hath himself pulled down the superstructure he had raised on it. Which, nevertheless, I do not believe he would have done had he but considered the whole matter more thoroughly. [...] But as for 
me, neither this nor any other difficulty shall have so great an influence on me as to make me renounce that which I know to be manifestly agreeable to reason [...]. For in the present case something peculiar lies hid, which [...] will, perhaps, hardly be discovered till such time as the manner of vision is more perfectly make known. Concerning which, I must own, I have hitherto been able to find out nothing that has the least shew of probability, not to mention certainty. I Shall, therefore, leave this knot to be untied by you, wishing you may have better success in it that I have had. (2013[1860], pp.152-33; NTV, §29)
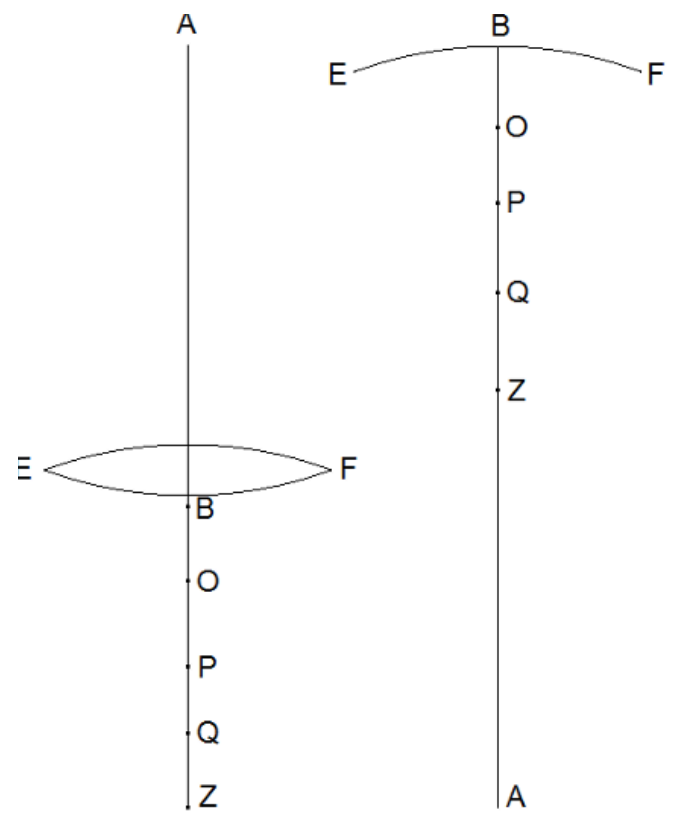

Figure 4: Barrow's problem

From this extensive quote, it is easy to infer that Barrow was a careful methodologist. He is not willing to abandon the basic principles of the geometrical theory in the face of the slightest difficulty. He believes it is reasonable to defend the hard core of his program, at least until he can count on a new perspective that offers better alternatives for the future.

What is the problem pointed out by Barrow? This case produces two anomalies for the geometrical theory of vision. (i) If we accept to $P_{5}$, then the observer must see the object as located behind the head, which would be unintelligible. (ii) It violates $P_{3}$, for according to this principle, the farther the object appears to the observer, the less is the divergence of the rays, and this does not occur in the Barrovian case. Now, why do these anomalies emerge? With the purpose of presenting this aspect 
in detail, we will first make a detour regarding $P_{3}$. Figure 5 shows a pair of eyes $O_{1}$ and $O_{2}$, with a punctual source of light $A$ and the paths of rays $A O_{1}$ and $A O_{2}$. As we had foreseen with the principle $P_{3}$ if $A$ is taken farther, the angle $O_{1} A O_{2}(\alpha)$ decreases; i.e., the divergence of the rays decreases. When $A$ is taken too far, the rays tend to approach the parallels drawn with dotted lines. In this case, angle $\alpha$ tends to $0^{\circ}$. There is no possibility - without the intervention of some optical instrument - that the rays coming from a punctual object arrive at the eyes through paths such as $B O_{1}$ and $C O_{2}$ (Figure 6). In that case, we would say that the object surpasses the least divergence possible. Nevertheless, if this situation occurs, we could think this is a case of anomalous divergence; it seems that the rays come from an object located behind the eyes. According to $P_{5}$, if the sensorium prolongs rays $B O_{1}$ and $C O_{2}$ to determine the location of the object (or the image of the object), the sensorium will judge that the rays come from a point located behind the head. This conclusion seems either surprising or absurd: in what sense do we say the mind judges the object as being located 'behind the head'? In this article, we will not concentrate on this last aspect of the Barrovian case; ${ }^{9}$ we will only focus on the anomaly that it generates for $P_{3}$, that is, the connection between the distance at which the object appears and the divergence of the rays entering the eye.

Figure 6 shows the virtual object $A^{\prime}$, an object that we can conceive as being behind the head. If the observer walks toward $A$ (real object), the greater the divergence between $A O_{1}$ and $A O_{2}$ will be (i.e., the greater the angle $\alpha$ ). If the observer moves toward $A^{\prime}$, we would be forced to admit that the divergence between $B O_{1}$ and $C O_{2}(\beta)$ is less (in the case it could be measured), for it is below the least divergence possible $\left(0^{\circ}\right)$ and the rays go beyond (or exceed) the parallels that establish the limit condition. ${ }^{10}$ When $A$ approaches from an infinite distance to the vicinity of the observer, $\alpha$ increases from $0^{\circ}$ to a maximum of $180^{\circ}$. Additionally, if $A^{\prime}$ approaches from an infinite distance to the location of the observer, $\beta$ decreases from $360^{\circ}$ to a minimum of $180^{\circ} .{ }^{11}$ The argument can be better understood if we consider Figure 7 . This figure presents the behavior of the divergence angle (which could be $\alpha$ or $\beta$ depending on the case) in function of the distance from the point where the prolongations of the rays converge to the observer $(D) .{ }^{12}$ When the approach is between the observer and $A^{\prime}$ (Figure 6), the divergence $(\beta)$ decreases; when the approach is between the observer and $A$, the divergence $(\beta)$ increases. For the moment, let us grant that even if the lines $B O_{1}$ and $C O_{2}$, which come from a certain source, cannot intersect in front of the observer, they can meet behind the observer (no matter how paradoxical or absurd it may sound). Given that angle $\beta$ decreases as $A^{\prime}$ approaches the observer, we will call the divergence between $B O_{1}$ and $C_{2}$ an anomalous divergence. Meanwhile, we will call the divergence $\alpha$ a standard divergence, provided that it increases as the object comes nearer and, moreover, that the rays come from a point in front of the observer. $^{13}$ 


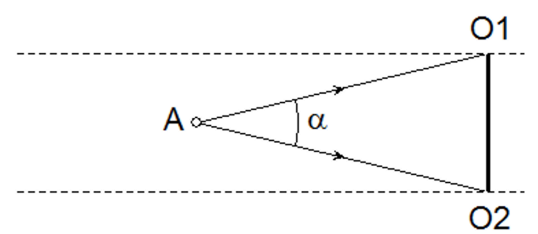

Figure 5: Divergence of rays of light [https://www.geogebra.org/m/gbpprc8c Dynamic points: $A$ and $\left.O_{1}\right]$

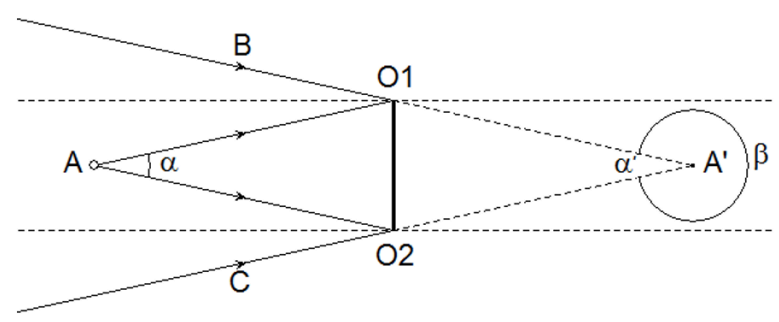

Figure 6: Anomalous divergence of rays of light [https://www.geogebra.org/m/jesac3md Dynamic points: $A$ and $\left.O_{1}\right]$

In our opinion, the main difficulties that emerge from the interpretation of the Barrovian Case and from the crucial aspect that Berkeley observes behind the anomaly are due to a lack of understanding of the difference between standard divergence and anomalous divergence.

We will present our view of the anomaly keeping in mind the distinction between anomalous and standard divergence that we have just suggested. In Barrow's problem, anomalous divergence results from the interposition of an optical instrument between the object and observer. We ask the reader to go to Figure 8, where $A$ represents the bottom of an object in front of a lens whose vertex is $B, Z$ is the location of the image of $A$ adjusted to the geometry of the lens, and $O_{1}$ and $O_{2}$ are the positions of the two eyes. ${ }^{14}$ The lines $A O_{1}$ and $A O_{2}$ (represented with dotted strokes) exhibit

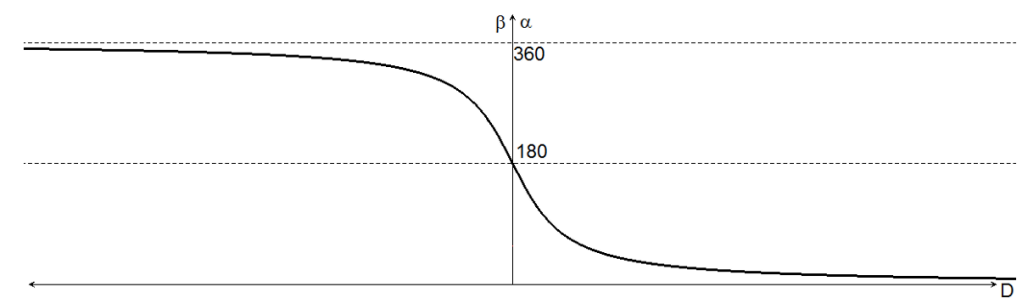

Figure 7: Standard and anomalous divergence depending on the distance from the observer 
the paths of light towards the observer provided there is no optical instrument in between. Furthermore, the angle $O_{1} A O_{2}(\alpha)$ measures the degree of standard divergence for the naked visual perception of the object. The paths $A C O_{1} Z$ and $A D O_{2} Z$ show the trajectories of the light that comes from $A$ and passes through the optical instrument; this instrument forces the rays of light to converge at $Z$ (according to principles $P_{1}$ and $P_{2}$ ) if they were prolonged with the aid of imagination. The angle $C Z D(\beta)$ measures the degree of anomalous divergence for the observation of the object through an optical instrument (principle $P_{5}$ ); $O, P$, and $Q$ represent some of the points where Barrow suggests the observer can be situated for the analysis.

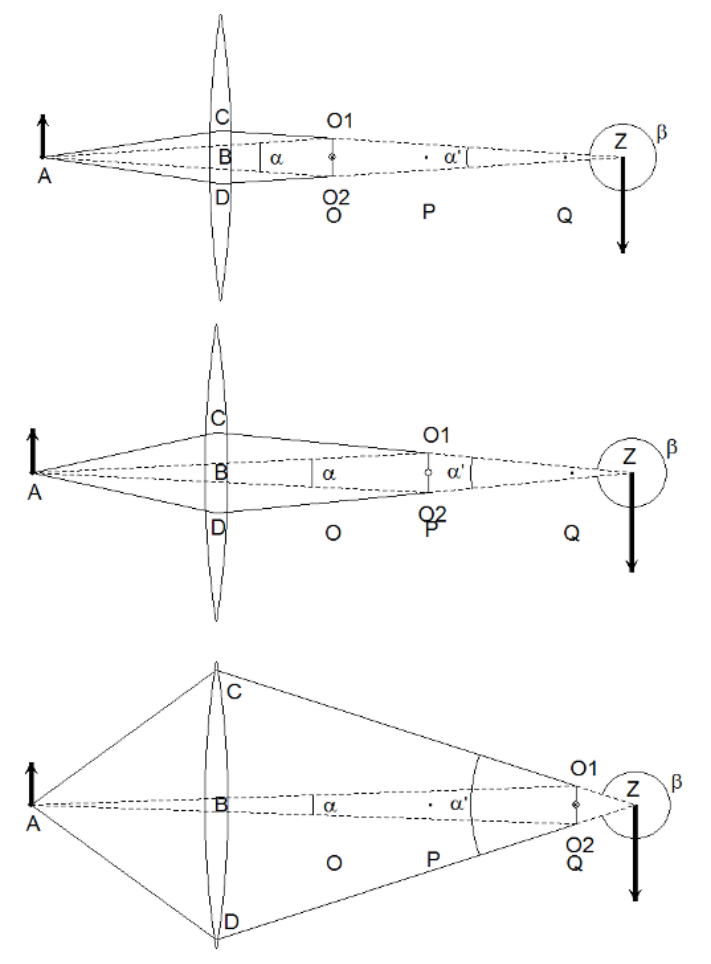

Figure 8: Barrow's problem (standard divergence vs anomalous divergence) [https://www. geogebra.org/m/pggyj4me Dynamic points: the pair of eyes by dragging the middle point]

As we said, we will omit the fact that $P_{5}$ suggests the strange result that the object must be seen behind the head of the observer. When the spectator is situated at $O$, the angles $\alpha$ and $\alpha^{\prime}$ are practically identical. In this case, the extension of the distance of the object perceived with the naked eye coincides with the extension of the distance of the imagined object when it is perceived through a lens. When the observer moves to $P$, the anomalous divergence decreases while $Z$ is perceived to be 
closer. ${ }^{15}$ This contradicts the corollary that follows from $P_{3}$; i.e., that if the divergence decreases, the object must appear as being farther away. When the observer arrives at a point very close to $Z$, let us say $Q$, the anomalous divergence decreases, and the object is seen so close that vision is no longer clear for the muscles of the eye are unable adjust sufficiently for the production of clear images. Keeping all of this in mind, we can synthesize Barrow's perplexity: according to the geometrical theory, when the divergence of the rays coming from the same point decreases, we expect the sensorium will judge that point (or object) as farther away; however, the above analysis shows that, in the case just mentioned, the object is perceived as closer while the divergence decreases. Figure 9 shows the same analysis for the case of an object seen via a concave mirror.

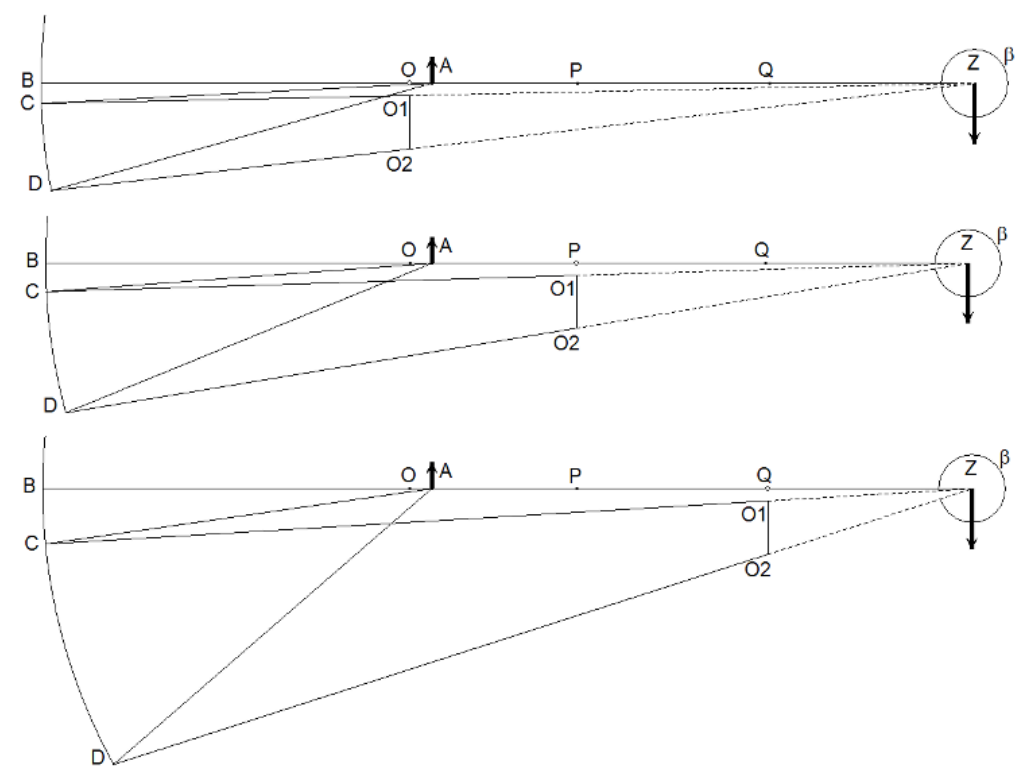

Figure 9: Barrow's problem (concave mirror) [https://www.geogebra.org/m/jke3jq7j. Dynamic points: the pair of eyes by dragging the projection onto the axis $]^{16}$

Barrow points out that the expectations deduced from the geometrical theory do not agree with what we have experienced. First, we do not see objects behind our heads. ${ }^{17}$ As Barrow himself suggests, according to the geometrical theory, the objects would have to appear as if they were "before the eye at a vast distance off, so great as should in some sort surpass all sensible distance". (NTV, §29). Objects would be seen in front and at a distance that exceeds the farthest objects that could in fact be in front of us. Second, we also hoped that, provided the least possible divergence $\left(0^{\circ}\right)$, the object would be located at an infinite distance. For that same reason, if the 
divergence could be even less (as it in fact is in the case of anomalous divergence), then the object would have to appear even farther than at an infinite distance. We could say, to enhance Barrow's perplexity, that objects should seem to be at a greater distance than infinity itself. Vasco Ronchi compares the expected perception with the perception that is effectively reported when an object is located in the vicinity of the focus of a concave mirror: "When the observer looks at the mirror, he should see virtual images infinitely distant; that is, he should see an endless hole behind the mirror, and at the back of this hole the image of the object should appear. No one has ever seen anything of the sort" (1991[1955], §192). ${ }^{18}$ Third, it is highly problematic that we contemplate an object whose divergence decreases as it comes closer. In Barrows words, "by how much the rays falling on the eye do more converge by so much the nearer doth the object seem to approach" (NTV, §29). Greater convergence equates, according to our categories, to smaller anomalous divergence. ${ }^{19}$

\section{Berkeley's solution}

How does Berkeley think we perceive distance? The perception of distance does not happen, as we have already said, immediately; we perceive distance through another idea that is itself immediately perceived. We have also seen that angles and lines are not among those ideas that are perceived immediately and from which we could infer the distance between an object and observer. Now, Berkeley does believe we are directly or immediately aware of tactile or kinesthetic sensations produced either by the movements of the eye or by the muscular actions that determine the necessary adjustments of the crystalline to assure the sharpest convergence on the retina (NTV §16). According to him, the subject judges the distance of an object with the help of associations between certain tactile impressions and certain visual impressions. The perceptions of our muscular efforts are among those proper sensibles of touch. Yet, if we adopt the heterogeneity thesis ${ }^{20}$ defended in NTV ( $\left.\$ \S 121-138\right)$, there is no way to establish a necessary bond between this tactile information and the so-called visual perception of distance. The link between the two is, in the philosopher's opinion, a contingent link that has been established in virtue of experience and repeated associations (NTV, §17). In addition to muscular actions, Berkeley also referred to the confusion of images as another idea that is, according to him, immediately perceived and that can be considered as a sign for distance. If, for the sake of discussion, we accept that the intervention of the mind begins once an image has been printed on the retina, the mind alone might recognize immediately if such a painting is sharp or blurry, or if it is weak in its illumination or not. Berkeley says:

An object placed at a certain distance from the eye, to which the breath of the pupil bears a considerable proportion, being made to approach, is seen 
more confusedly: and the nearer it is brought the more confused appearance it makes. And this being found constantly to be so, there ariseth in the mind an habitual connexion between the several degrees of confusion and distance; the greater confusion still implying the lesser distance, and the lesser confusion the greater distance of the object. (NTV, §21)

No man, I believe, will pretend to see or feel those imaginary angles that the rays are supposed to form according to their various inclinations on his eye. But he cannot choose seeing whether the object appear more or less confused. It is therefore a manifest consequence from what hath been demonstrated, that instead of the greater or lesser divergency of the rays, the mind makes use of the greater or lesser confusedness of the appearance, thereby to determine the apparent place of an object. (NTV, §22)

Berkeley's suggestion has several assumptions. (i) We must imagine that there is a primitive recognition of a certain objective distance that, although it cannot be perceived by sight, must be taken as given for us in order to talk (in an objective way) of a body that moves closer or farther. This primitive sense of distance can be apprehended through tactile information, and moreover, through de perception of the movements of our body $(N T V, \S \S 45,50,99)$. Berkeley, in $\S 55$, explains why tactile information is primary and why it can give us an account of this "objective distance":

The magnitude of the object which exists without the mind, and is at a distance, continues always invariably the same: but the visible object still changing as you approach to, or recede from the tangible object, it hath no fixed and determinate greatness. Whenever therefore, we speak of the magnitude of anything, for instance a tree or a house, we must mean the tangible magnitude, otherwise there can be nothing steady and free from ambiguity spoken of it. (NTV, §55).

Given that the distance and size perceived by touch remain constant for any observer while the visual do not, tactile distance can be considered as objective: there are objects outside the mind (if we focus solely on Berkeley's stance in the NTV) that are at a distance from us, and touch is what gives us knowledge of it. (ii) Berkeley believes that the contemplation of confusedness (blurriness) relating to an image when we fail to ensure a precise convergence of the light rays in the retina. Hence, even if we do not perceive rays arriving at the back of the eye, Berkeley does think we can recognize the confusion that covaries with the blurriness of the painting printed in the retina. $^{21}$ (iii) Berkeley's argument demands we concede there is a range of muscular reactions so that, when an object comes too close (when an object almost touches our eyes), certain muscular efforts that exceed this range are required to ensure a clear painting on the retina; for that reason, the sensory apparatus would never be able to make a clear image of the object. Finally, (iv) it is assumed that confusion 
(blurriness) covaries with a failure to ensure a precise convergence of light rays on the retina. This confusion would disappear if we allow the necessary adjustments in the crystalline. Thus, the description given by Berkeley in the passage we have quoted must be understood in the following terms: if the visual apparatus is adjusted for receiving the pictoric image of a distant object on the retina, and we move this object closer without making new adjustments in the crystalline, the printed image on the retina will be blurry - because of the lack of focus - and it would be even more blurry if we move the object even closer. This failure of the adjustments in the crystalline is correlated with the perception of a blurry image.

Berkeley refuses to regard the sensorium as paying attention to angles or rays when it judges distance. The Irish philosopher prefers to think of the sensorium as perceiving immediately an image that covaries with the physical painting printed on the retina. Assuming this is the case, we judge an object as being near, not by sensing the great divergence of the light rays that arrive at the eyes (or at just one eye), but by noticing the irremediable confusion of the painting perceived. This is one of the aspects of Berkeley's proposal that is supposed to replace $P_{3}$ and $P_{5}$. It is important to keep in mind that there is no necessary connection between the blurriness of the image and objective distance. The link, in this case, is a contingent association fed by habit, custom, practice, and failure. Berkeley even came to think of the possibility of considering the relationship between distance and confusion as occasional causes in the terms of Malebranche (NTV, §§25, 28).

How does Berkeley solve Barrow's problem? Berkeley regarded the failure of the geometrical theory in facing Barrow's difficulty as an opportunity to show the superiority of his own theory. Berkeley, before engaging on his own account, explained how we ought to understand the sources of clear and unclear vision. The philosopher repeated the account and the figure used by Molyneux in his Dioptrica Nova. ${ }^{22}$ Berkeley presents the account as follows: "any radiating point is then distinctly seen when the rays proceeding from it are, by the refractive power of the crystalline, accurately reunited in the retina or fund of the eye: but if they are reunited, either before they arrive at the retina, or after they have passed it, then there is confused vision". (NTV, §34). Some lines later, the author insists: "Confused vision is when the rays proceeding from each distinct point on the retina, but take up some space thereon, so that rays from different points become mixed and confused together" (NTV, §35). Additionally, "[f]aint vision is when by reason of the distance of the object or grossness of the interjacent medium few rays arrive from the object to the eye". (NTV, §35).$^{23}$

Berkeley explained his proposal using Figure 10. The crystalline, as shown in part 1 , is adjusted to ensure that the rays arriving in a parallel way converge on precisely one point on the retina. The painting, in this case, must be perceived with no confusion. This is what happens when we are observing a very distant source of light (where the divergence of the rays is a minimum). If the rays from each extreme 


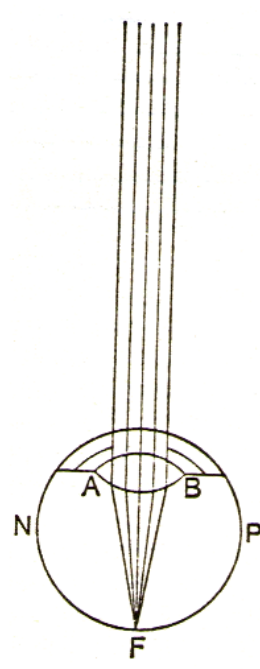

1

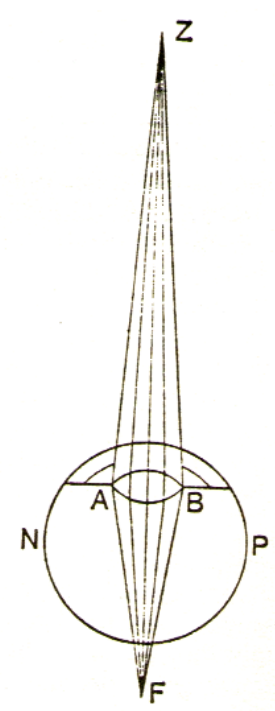

2

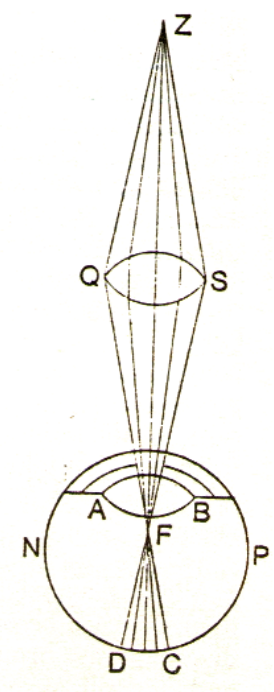

3

Figure 10: Comparison between clear and confused paintings. NTV, §35

coming from $Z$ enter the eye with a divergence different from $0^{\circ}$ and we do not allow an adjustment of the crystalline as in part 1 , they reunite at a point that is beyond the retina (point $F$ of part 2). In this case, the painting printed in the retina is no longer reduced to a point but has the shape of a circle and thus has a certain degree of confusion. Similarly, as in part 3 of the figure, if, using an optical instrument $Q S$, we manage to make the rays coming from $Z$ enter with an anomalous divergence and converge at a point $F$ before the retina, the painting will be just as blurry, for the rays would cover the area $D C$ on the retina. The interesting thing about this case is that in both parts 2 and 3, the painting on the retina is unclear. Thus, by only observing the painting, we would not know if the confusion is caused by an anomalous divergence or a standard divergence. In other words, there is an indetermination over which could be the cause of the blurriness.

Hence, when facing a blurred painting covering the area $D C$ on the retina, the mind would naturally judge the object as being near (if it ignores other circumstances). Nevertheless, if the subject is in a situation where he knows there could be a lens interfering between the object and his eyes, he would have to hesitate in deciding whether the confusion is due to a standard divergence (2) or anomalous divergence (3). This would imply that we would not know how to interpret the blurriness of the image, if in a standard way (i.e., as meaning closeness) or if in an anomalous way (i.e., as meaning long distance). We would therefore need additional 
information to judge distance correctly. According to Berkeley, if we have certainty that there is an intervention of an instrument such as QS (which we could know from tactile information of the existence of such an instrument), the mind could change the usual interpretation (or the usual association):

The eye, or (to speak truly) the mind perceiving only the confusion itself, without ever considering the cause from which it proceeds, doth constantly annex the same degree of distance to the same degree of confusion. Whether that confusion be occasioned by converging or by diverging rays, it matters not. Whence it follows, that the eye, viewing the object $Z$ through the glass $Q S$ (which by refraction causeth the rays $Z Q, Z S$, etc., to converge) should judge it to be at such nearness, at which if it were placed, it would radiate on the eye with rays diverging to that degree, as would produce the same confusion which is now produced by converging rays, i.e. would cover a portion of the retina equal to $D C$. (NTV, §36)

\section{Assessment of Berkeley's answer to the anomaly}

Has Berkeley explained a difficulty that was definitely impossible for the geometrical theory to face? If the answer is in the affirmative, then the Barrovian Case can be regarded as a crucial anomaly that rules in favor of Berkeley and forces one to abandon or to make serious adjustments to the geometrical theory. However, in our opinion, the explanation of the Irish philosopher offers his opponent the clues necessary to solve the anomaly using precisely Berkeley's same strategy. Thus, Barrow's case does not rule in favor of Berkeley. Both Berkeley and his opponent have to deal with this case as an anomaly. In Berkeley's situation, the anomaly is associated with an indeterminacy: a blurry image can be interpreted (or be associated with tactile experience) in two ways. On the one hand, we can make a standard association. In such an interpretation (part 2 in Figure 10), light rays from a source of light in front of the observer enter the eye in a divergent way and converge later when passing through the crystalline; in these circumstances, the point of convergence is located beyond the retina. On the other hand, we can make an anomalous association. Here (part 3 in Figure 10), the rays of light arrive at the eye in a convergent way and, after passing through the crystalline, arrive at the retina in a divergent way. Keeping this in mind, Berkeley invites us to make a shift of convention when we have in our hands additional information about the intervention of some optical instrument that could be generating an anomalous divergence: "Whereas heretofore the greater confusions were always wont to signify nearer distances, they have in this case a direct, contrary signification, being connected with the greater distances" (NTV, §32). In that order of ideas, an observer can discover different regularities and establish different associations in order to change the meanings or conventions assigned to 
greater or lesser confusedness of the observed images. In our view, the defender of the geometrical theory could also appeal to a shift of convention to face the different interpretations connected to the reading of the angles of the rays when they enter the eye. The mind can posit, in virtue of the standard divergence $\alpha$ (Figure 5), the existence of a source of light at a distance $D$ in front of the observer; or it can posit, in virtue of an anomalous divergence $\beta$ (Figure 6 ), the convergence point at a distance $D$ behind the observer.

If we admit either the principles of the geometrical theory or the principles from Berkeley's theory, we would have to assume that the mind proceeds to evaluate the information at hand in anticipating future reactions of the observer. In the case of the geometrical theory, what is evaluated are the angles the rays form when arriving at the eyes; in the second case, it is the confusion or the clarity of the mental image that covaries with the painting printed on the retina. Now, the assessment of visual distance is a complex process that cannot be reduced to the assumptions of the geometrical theory or the assumptions of a semiotic theory. This evaluation implies, additionally, physiological, environmental, and psychological factors. Whichever way these factors are organized or connected, the mind construes an object that is always located in front of the observer. ${ }^{24}$ Judging the distance or location is thus not a task that is carried out in the physical space; it is either a projection or an association made by the mind. As we have seen, in the moment an anomalous situation is produced, the observer cannot depend on the general associations he had always done. In both scenarios (both Berkeley's and the geometrical theorist's) the observer needs to relearn and to reinterpret the clues for judging distance. In addition to this, the observer requires information of the external circumstances that are modifying normal perception and, moreover, a precise knowledge of how these circumstances affect her vision. If Berkeley's observer is allowed to reinterpret and change the usual associations, why is the geometrical theorist not? The Barrovian case, then, proves that there is not necessary connection between the size of the angles and the perception of distance, just as there is not a necessary connection between the blurriness of the image and the perception of distance. More information is required. But this does not entail, in itself, that we do not use geometrical clues for judging distance; it only entails that the meanings we ascribe to these clues can change depending on the situation.

We could then change the graph in Figure 7 to regard in a better way the absolute value of distance. Figure 11 shows the adjustments and lets us see clearer that the distance decreases when the anomalous divergence decreases or when the standard divergence increases.

If we had a way to measure the extent of confusion of a painting in order to envision the behavior regarding distance, this graph would have to show the same sort of indetermination exhibited in Figure 11; i.e., that for a given distance, we would not 


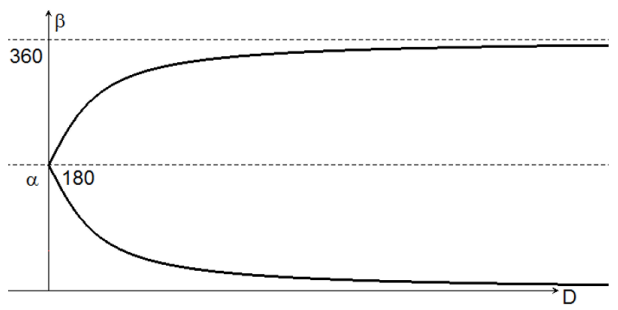

Figure 11: Absolute value of distance vs standard and anomalous divergence.

be able - unless we had additional information - to ensure the confusion is due to a standard association (or standard confusion) or to an anomalous association (or anomalous confusion).

Berkeley, and Barrow himself, notice that the difficulty presents a violation of principles P3 and P5; there seems to be a problem in the way the geometrical theory gives an account of how we judge distance and how we determine the location of images of an object. Nevertheless, as we have already anticipated, we consider that the anomaly can be faced if we suggest a restatement (following Berkeley's strategy) of the basic corollary that follows from the third principle:

An object $A$ is perceived closer than another object $B$ if one of the following conditions is given: (i) the standard divergence caused by $A$ is bigger than the standard divergence caused by $B$; (ii) the anomalous divergence caused by the interposition of an optical instrument which produces a virtual convergence in $A$ is smaller than the anomalous divergence which produces a virtual convergence in $B .{ }^{25}$

Therefore, no difficulty emerges for the theorist of the geometrical theory if we give her the license to change the convention when she is aware of whether the divergence is standard or anomalous. The divergence is standard if the rays entering the eye come from a point in front of the observer and the divergence is anomalous if the prolongations of the rays converge at a point behind the observer. In any case, the mind projects an object in front.

As we have already pointed out, the explanation offered by Berkeley also admits a shift of convention when the observer notices the presence of an optical instrument that changes the standard divergence into an anomalous divergence. Berkeley compares the perplexity that an English speaker would experience if he or she encounters a foreign speaker who uses the same words but with a completely different meaning. The difficulty could vanish if the speaker finds a way to re-signify the foreign speaker's words. We will quote the way Berkeley introduces this comparison. We will then suggest a way of paraphrasing this passage to show how the defender of the 
geometrical theory could also appeal to a shift of convention to face the anomaly. The passage can be read with complete sense under the interpretation of Berkeley's theory or under the interpretation that a theorist of the geometrical theory could offer if we allow him or her to introduce a change of convention. Berkeley's passage is:

This case is much the same as if we should suppose an Englishman to meet a foreigner who used the same words with the English, but in a direct contrary signification. The Englishman would not fail to make a wrong judgment of the ideas annexed to those sounds in the mind of him that used them. Just so, in the present case the object speaks (if I may so say) with words that the eye is well acquainted with, that is, confusions of appearance; but whereas heretofore, the greater confusions were always wont to signify nearer distances, they have in this case a direct, contrary signification, being connected with greater distance. Whence it follows that the eye must unavoidably be mistaken, since it will take the confusions in the sense it has been used to, which is directly opposed to the true. (NTV, §32)

We suggest the following rephrasing (the changes are written in italics):

Just so, in the present case, the object causally interacts (if I may so say) producing modifications that the eye is well acquainted with, that is, with impressions that could be read in terms of greater or less divergence of the rays that enter the eye; but whereas heretofore the greater divergence was always wont to signify nearer distances, it has in this case a direct, contrary signification, being connected with greater distances. Whence if follows, that the eye must unavoidably be mistaken, since it will take the degrees of the divergence in the sense it has been used to, which is directly opposed to the true.

C. M. Turbayne follows carefully the passages that we have quoted $(\S \S 32,36)$ to defend that the Irish philosopher is able to explain Barrow's anomaly while the geometrical paradigm of vision is unable to do so (1962, pp.172-85). The author says "Now the Barrovian Case is exactly like this double-talk. It involves not a new language with new terms but an old language with new meanings that have to be re-learned" (1962, pp.17-178). The rephrasing that we have suggested shows that aphorism §32 admits a dual interpretation: we can exchange some of the expressions of Berkeley's theory for adequate geometrical expressions and still obtain a meaningful statement that can be regarded as a correct description of the difficulty using the assumptions of the geometrical theory. Just as Berkeley's Englishman is capable of reinterpreting the words of the English language, an observer can also change the meanings of the angles of the rays entering the eye. For example, we can imagine a subject who is asked to wear a pair of glasses with lenses that produce the same effects as the lenses in Barrow's case. Assuming that the subject judges distance by 
perceiving angles, he will be forced to reinterpret the association between the angles and distance of the object. As long as he is aware of the effects produced by the glasses, he can associate a greater angle with a larger distance and a smaller angle with a smaller distance. We do not see how Turbayne could answer this criticism.

T. M. Lennon, unlike Turbayne, believes that Barrow's case cannot be taken as a crucial anomaly. His defense is anchored on two assumptions. The first consists of reading Berkeley's arguments in his NTV as arguments clearly oriented towards a defense of the idealism implicit in the immaterialism from the Principles. In our opinion, we can obtain the same result as Lennon without saying that NTV is an introduction to immaterialism. Regardless of whether the NTV is an introduction to immaterialism (which we are not denying), the Barrovian case (as presented by Berkeley) is not necessarily a crucial anomaly that disconfirms the geometrical theory. The second consists of clearly defining what is the immediate object of our visual perception: (a) are they images or physical paintings that can be taken as representations of the objects themselves? (representational realism); (b) are they images or mental paintings that can be identified with things perceived? (immaterialist idealism); or (c) are they physical objects that can be immediately contemplated? (direct realism). Our strategy for not seeing the Barrovian Case as a crucial anomaly does not require us to first elucidate what is to be taken as the immediate object of visual perception.

The next passage shows that Berkeley acknowledges that Barrow's mistake is due to pretending to extend an analogy towards cases that demand, rather, a change of convention, just as we have suggested:

It having been observed that the most diverging rays brought into the mind the idea of nearest distance, and that still, as the divergency decreased, the distance increased: and it being thought the connexion between the various degrees of divergency and distance was immediate; this naturally leads one to conclude, from an ill-grounded analogy, that converging rays shall make an object appear at an immense distance: and that, as the convergency increases, the distance (if it were possible) should do so likewise. That this was the cause of Dr. Barrow's mistake is evident from his own words which we have quoted. Whereas had the learned doctor observed that diverging and converging rays, how opposite soever they may seem, do nevertheless agree in producing the same effect, to wit, confusedness of vision, greater degrees whereof are produced indifferently, either as the divergency or convergency of the rays increaseth. And that it is by this effect, which is the same in both, that either the divergency or convergency is perceived by the eye; I say, had he but considered this, it is certain he would have made a quite contrary judgment, and rightly concluded that those rays which fall on the eye with greater degrees of convergency should make the object from whence they proceed appear by so much the nearer. (NTV, §39)

If the dual interpretation of the cited passage is valid, then Barrow's problem 
does not necessarily force us to give up the geometrical theory. Berkeley has not proved that it is impossible to use the principles of the tradition to solve the difficulty. These principles still allow us to make correct inferences about distance if we allow the introduction of additional information on the circumstances. One could think of someone who insists on judging distance through a geometrical calculus; the only difference with the Barrovian case is that the way you make those judgements must change if you have all the information at hand. However, Berkeley demands that information in a similar way. In that order of ideas, if the analysis we have offered is correct, Barrow's problem cannot be properly regarded as a crucial anomaly that definitively rules against the geometrical theory. Nevertheless, the geometrical theory must explain why the observer believes she is seeing an object in front her while she would have to judge it as being behind her. It is not easy to envisage a way to give an account of this phenomenon. Throughout this article, we have not pretended to present a defense in favor of the geometrical theory. For us, its assumptions do not lead us to a full description of visual perception and, additionally, they assume a passive role for the observer. What we have wanted to show is that Berkeley's arguments that invoke the superiority of his theory using the Barrovian Case cannot be taken as completely convincing against the geometrical theory.

\section{Objection from an anonymous reviewer}

The objection we received (and for which we are thankful) is based on the fact that the objects we observe in our visual field always appear as if they were in front of us, never behind our visual system. This phenomenon occurs in the Barrovian Case even though $P_{5}$ indicates that the rays coming from the object and entering the eye seem to diverge from a point located at the back of our head. The anonymous reviewer asks: "Instead of making light rays to continue in imaginary lines to a point behind the head, would it not be easier to use a different ad hoc hypothesis to defend the geometric theory? If the angles made by light rays at the pupil or at the eyes are greater than right angles, then we see objects as if they were placed in two imaginary lines that are symmetrical to the converging light rays relative to lines that fall perpendicular on the pupil or eyes".

Indeed, despite the fact that $P_{5}$, in the Barrovian case, suggests that we should perceive a source of light coming from the back of our head, the mind always projects an effigy that we believe we contemplate in front of us. The reviewer suggests an interesting ad hoc hypothesis to reestablish frontal vision. Let us see the subject carefully. The two eyes $O_{1}$ and $O_{2}$ from an observer contemplate a source of light $A$ on front (Figure 12). The paths of light are modified because of the interference of an optical instrument between the object and the observer. The vectors show the new 
paths of two outer rays that, if it had not been for the intervention of the instrument, would have arrived directly to $O_{1}$ and $O_{2}$ respectively. The eyes look towards the side where $A$ is located. The use of $P_{5}$, without any changes, leads us to expect that the observer must admit that he is observing a source of light that seems to come from $Z$ (behind the head). Given that the result is shocking, the reviewer proposes an ad hoc modification for $P_{5}$ for these cases. Since each ray, $E O_{1}$ and $E O_{2}$, arrives at the corresponding eye and forms an angle greater than $90^{\circ}$ with the line $O_{1} O_{2}$, it is suggested to draw two imaginary lines: one symmetrical to $E O_{1}$ relative to $O_{1} D$, and another line symmetrical to $\mathrm{BO}_{2}$ relative to $\mathrm{O}_{2} \mathrm{C}$. The angles $\mathrm{CO}_{2} \mathrm{O}_{1}$ and $\mathrm{DO}_{1} \mathrm{O}_{2}$ are right. The source of light would be seen in the intersection of these two auxiliary constructions, i.e. $Z^{\prime}{ }^{26}$ Therefore, though $P_{5}$ establishes that the observer sees a source of light at $Z$ (behind the head) under the anomalous divergence $\beta$, the modification ad hoc demands that the observer sees a source of light at $Z^{\prime}$ (in front) under a standard divergence $\alpha$. $Z$ and $Z^{\prime}$ are specular images in relation to $O_{1} O_{2}$. Hence, an increase in $\beta$ (that is obtained if the observer moves farther from $Z$ and consequently from $Z^{\prime}$ ) corresponds with a decrease in $\alpha$. This way, the initial corollary according to which a decrease in the divergence covaries with an increase in the distance between the virtual source and the observer is reestablished.

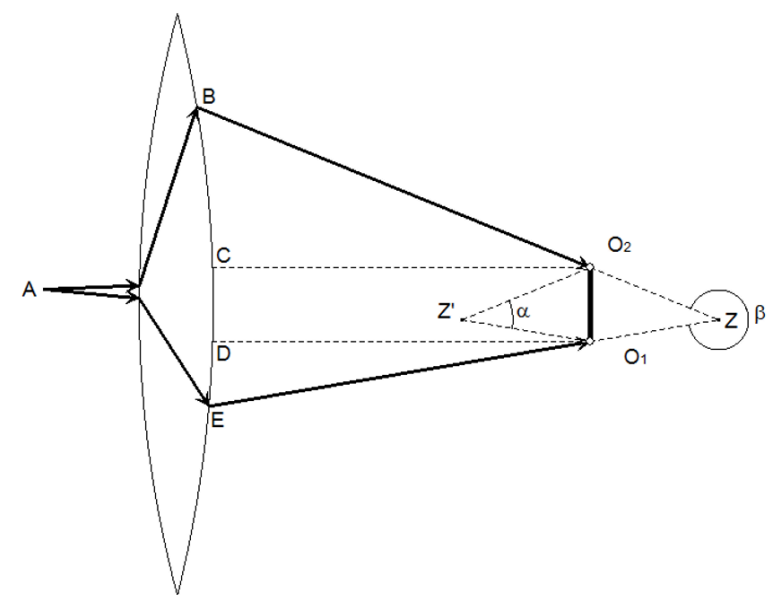

Figure 12: Ad hoc hypothesis to reestablish the location in front

The reviewer concludes that "This revision of the geometric theory seems to me to be more economical than the construction of the authors". Nevertheless, we have two purposes in our paper. First, we want to show that there are ways to save the geometrical theory from the anomaly presented by Barrow. We do not expect to find, among the possibilities, the most economical or simple. In fact, we still don't see how this last solution is more economical than ours. Second, we want to adopt the same 
strategy used by Berkeley, that is, to suggest a shift of convention (or meaning). With the ad hoc changes to $P_{5}$ the reviewer does not need to change the convention that associates lesser divergence with greater distance. We instead left $P_{5}$ unchanged and introduced a shift of convention that allows a minor modification of the corollary entailed by $P_{3}$. We have built an argument that follows the same line of explanation offered by Berkeley. This way, we show that the Barrovian Case does not offer a situation that allows us to decide between two rival theoretical approaches and, moreover, we use the same argumentative strategy suggested by Berkeley in order to face the anomaly.

\section{References}

Armstrong, D. M. 1960. Berkeley's Theory of Vision. Melbourne: Melbourne University Press. Atherton, M. 1990. Berkeley's Revolution in Vision. Ithaca: Cornell University Press.

Barrow, I. 2013[1860]. The Mathematical Works of Isaac Barrow. Cambridge: Cambridge University Press.

Berkeley, G. 1975[1710]. A Treatise concerning the Principles of Human Knowledge [PHK]. In: Philosophical Works, pp.71-153. London: Everyman.

Berkeley, G. 1975a[1709]. An Essay Toward a New Theory of Vision. In: Philosophical Works [NTV], pp.71-153. London: Everyman.

Berkeley, G. 1975b[1707-8]. Philosophical Commentaries. In: Philosophical Works, pp.305412. London: Everyman.

Berkeley, G. 1975c[1733]. The Theory of Vision Vindicated and Explained. In: Philosophical Works [TVV], pp.277-304. London: Everyman.

Helmholtz, H. von. 2005[1909]. Treatise on Physiological Optics ( $3^{\text {rd }}$ ed.). Trans. James P. C. Southhall. Mineola: Dover Publications, Inc.

Kepler, J. 2000[1604]. Optics: Paralipomena to Witelo \& Optical Part of Astronomy. Trans. William H. Donahue. Santa Fe: Green Lion Press.

Lennon, T. M. 2007. The significance of the Barrovian Case. Studies in History and Philosophy of Science 38: 36-55.

Luce, A. A. 1934. Berkeley and Malebranche, A Study in the Origins of Berkeley's Thought. London: Oxford University Press.

Molyneux, W. 1709. Dioptrica Nova. A Treatise of Dioptricks, in two parts. London: Benj.

Pitcher, G. 1977. Berkeley. London: Routledge.

Ronchi, V. 1991[1955]. Optics. The Science of Vision. Trans. Edward Rosen. New York: Dover Publications, INC.

Shapiro, A. 1990. The Optical Lectures and the foundations of the theory of optical imagery. In: M. Feingold (ed.) Before Newton, The life and times of Isaac Barrow, pp.105-78. Cambridge: Cambridge University Press.

Stack, G. J. 1991. Berkeley's Analysis of Perception. New York: Peter Lang.

Tipton, I. C. 1974. Berkeley, the Philosophy of Immaterialism. London: Methuen \& Co Ltd.

Turbayne, C. M. 1962. The Myth of Metaphor. New Haven: Yale University Press. 


\section{Notes}

${ }^{1}$ Throughout the article, we will use the following abbreviations for some of Berkeley's works: NTV for Essay towards a New Theory of Vision; TVV for Theory of Vision Vindicated; PHK for A Treatise Concerning the Principles of Human Knowledge.

${ }^{2}$ Berkeley's differences with Newton's distinctions between absolute and relative spacetime anticipated the criticisms made by thinkers such as Mach, Hertz, and Einstein. His refusal to accept the existence of infinitesimal magnitudes also anticipated the revisions that later mathematicians (such as Cauchy, Bolzano, and Weierstrass) elaborated for the foundations of calculus.

${ }^{3} N T V$ and TVV differ in their structure. NTV, analytically, first destroys false assumptions to make a way for truth. TVV follows the reverse order; i.e., it first stablishes the conclusions arrived at in the NTV as principles from which the rest of our knowledge can then be derived synthetically.

${ }^{4}$ To be more precise, Kepler recommended the term "paintings" (2000[1604], p.181).

${ }^{5} \mathrm{~V}$. gr.: the size of the object, the location of the object in relation to the instrument, the number of rays, the extension of the dispersion of the incident rays, and the geometrical properties of the optical instrument.

${ }^{6}$ Some of the figures will be accompanied by models made with the dynamic geometry software GeoGebra. Next to the figures' captions there will be the link and the specifications of the points the reader can move.

${ }^{7}$ Kepler arrived at this principle after showing the limitations of the classical method ascribed to Ptolemy. The Greek Mathematician conceived the location of the image as the point of intersection of the prolongation of the reflected ray with the perpendicular line to the mirror (or lens) drawn from the object.

${ }^{8}$ For the case that we are interested in studying, it is irrelevant whether perception is with one eye or two eyes.

${ }^{9}$ Why we see objects as always being in front of us is still a riddle for both Berkeley and the geometrical theory. Moreover, there's still a sense in which we do see objects as being behind us (for example, a rearview mirror). (See note 17).

${ }^{10}$ When we say the divergence between $\mathrm{BO}_{1}$ and $\mathrm{CO}_{2}$ is less, we are alluding to the convergence that Barrow seemed to identify: "Nay, it is even certain that by how much the rays falling on the eye do more converge by so much the nearer doth the object seem to approach". (NTV, §29).

${ }^{11}$ If the angle $O_{2} A^{\prime} O_{1}(\alpha)$ is taken in the opposite direction of the clock and assigned a positive magnitude, the angle $O_{2} A O_{1}\left(\alpha^{\prime}\right)$ has to be regarded in the same direction as the clock and must be assigned a negative magnitude. To make the presentation easier, we will assume $\beta=360^{\circ}+\alpha^{\prime}$ (keeping in mind that $\alpha^{\prime}$ has a negative magnitude).

${ }^{12}$ The function has the form $\alpha=2 \arctan \left(\frac{d / 2}{D}\right)$ where $d$ is the distance between the two eyes and $D$ is the distance from $A$ (or $A^{\prime}$ ) to the mid-point between the eyes.

${ }^{13}$ In order to avoid misunderstandings, it is important to clarify that the adjectives "anomalous" and "standard" are not descriptions of the divergence, for this would make it seem as if in each case we have a different type of angle. "Anomalous" and "standard" are assessments of the situations in which the observer is evaluating and interpreting the divergence. 
${ }^{14} \mathrm{O}_{1} A O_{2}$ can also represent a beam of light emanating from $A$ and entering the pupil. A. Shapiro made a thorough analysis of the difference between using $P_{5}$ considering two eyes or just one eye. The author believes that Kepler wrongly regards them as equivalent in all cases (1990, pp.119-27). The analysis in this article is not affected by Shapiro's remarks.

${ }^{15}$ The argument will be clearer if the reader focuses on the behavior of angle $\beta$ in the graph of Figure 7.

${ }^{16}$ In the figure, we omit rays that describe the perception of the object without mediation of the mirror.

${ }^{17}$ However, the anomaly may not appear as that problematic, for there are circumstances where we could intelligibly say we see objects behind our heads (e.g., when a driver sees objects behind him through a rearview mirror) or even behind the crystalline (e.g., entoptic objects in the vitreous humor). Lennon criticizes the commentators for presenting the difficulty as unsolvable (2007, p.47).

${ }^{18}$ Ronchi hopes the object's image is seen at the back of the presumed hole, for that is where the object must appear as farther than the greatest interval possible.

${ }^{19}$ Ronchi concludes that every anomaly in the perception of images via concave mirrors or lenses should force us to abandon $P_{5}$ : "seventeenth-century optics has shown itself to be utterly inadequate to explain the experimental data seen by anyone looking at a concave mirror. The fundamental hypothesis that "the eye sees a luminous point at the center of curvature of the waves reaching it" is almost never confirmed" (1991[1955], p.201).

${ }^{20}$ The heterogeneity thesis establishes that there are no common sensibles: the ideas of vision and the ideas of touch are qualitatively different (i.e., they share no characteristic whatsoever) and they do not refer to the same thing. For this reason, there are no necessary links between ideas of different senses.

${ }^{21}$ Berkeley's suggestion can be considered as problematic for several reasons. The author, throughout his essay, insists that the only proper sensibles of vision are light and color; everything we believe or say we see must be inferred solely from this information. However, there is nothing in light or color that allows us to deduce the idea of the confusion of images. Moreover, in our view, to be aware of the sharpness or the confusion of the image, we require a criterion for individuating objects in our visual field. If we limit ourselves merely to the pictoric information gathered in the retina and do not have at hand an accurate individuation (or distinction) criterion, we would not know what a sharp or confused image is like. For, how could I recognize if I am contemplating a painting with sharp boundaries in a confused way, or a painting with blurry boundaries in a clear way? If we had expectations before observing the painting, we could have an answer to the question; if that is not the case, then we cannot see a way to avoid the problem. It may seem strange to demand a criterion of individuation in order to identify when an image is blurry or clear. However, we believe individuation and recognition of clearness go together, for we can identify blurry limits only after we recognize the sort of objects we have in front of us and what the limits of those objects are.

${ }^{22}$ See the figure in Table 25 of Dioptrica Nova.

${ }^{23}$ In Molyneux's text: "Distinct Vision is then caused, when the Pencils of Rays from each point of an Object do accurately determine in Correspondent Points of the Retina. Confused Vision on the contrary, when these Pencils do intermix one with another. But Clear Vision is only caused by a Great Quantity of Rays in the same Pencil, illuminating the Correspondent Points of the Image strongly and vigorously. Faint Vision is then when a Few Rays make up 
one Pencil" (1709, p.109).

${ }^{24}$ Ronchi uses the term effigy to refer to these mental productions: "I attribute to the mind $[\ldots]$ the faculty of creating effigies [...]. When the mind has created the effigies, and located them in front of the eyes, the ego says that it sees the objects of the external world." (1957/1991, §89).

${ }^{25}$ The first case consists of a comparison between two sources of light that are supposed to be located in front of the observer. The second case compares two virtual points of convergence that are supposed to be behind the observer.

${ }^{26}$ In the second half of the $19^{\text {th }}$ century, German physiologist Hermann von Helmholtz, using the principles of the geometrical theory and proposals similar to Berkeley's semiotic approaches, argued that the allegedly external location of the source of light that causally triggers visual perception must be found in the intersection of two lines that consciousness projects outside in virtue of an unconscious inductive inference. These two lines, one for each eye, link the activation points on the retina (which are associated to the same source) with the nodal point of the corresponding eye. The nodal point for each eye is located on the optical axis and in the vicinity of the posterior face of the crystalline; this position can slightly change according to the adjustments of focus made by the crystalline (cfr. Helmholtz, 2005[1909], vol. 1, §§9-10; vol. 3, §26).

\section{Acknowledgments}

We are grateful to the Direction of Research and Innovation of Universidad del Rosario for the support given to the development of this research. 